\title{
A collision resolution algorithm for ad-hoc wireless LANs
}

\author{
D.V. Cortizo*, J. García**
}

*Electronic and Systems Department, University of A Coruna

Campus de Elviña s/n 15071 A Coruña, Spain, phone: +34.81.167150, fax:+34.81.167160,e-mail:dcortizo@des.fi.udc.es

**Computer Architecture Dep., Polytechnic University of Catalonia Jordi Girona 1-3, Campus Nord Mòdul D6 08034 Barcelona, Spain, phone: +34.3.401.6798, fax: +34.3.401.7055, e-mail:jorge@ac.upc.es

\begin{abstract}
In this paper* we propose and analyze a Medium Access Control (MAC) protocol for high-speed ad-hoc Wireless Local Area Networks. The MAC protocol is a collision resolution algorithm and aims to approximate the delay characteristics of an ideal multiplexer with FIFO discipline. The protocol groups the packets arriving in given time intervals. Packets belonging to the same set will be transmitted via a CSMA algorithm whereas the different sets will be served following a FIFO discipline. The results obtained by means of simulation and analytical models show that the protocol can support real-time services even when the network load is moderately high.
\end{abstract}

\section{Keywords}

Wireless LANs, Ad-hoc networks, Medium Access Control algorithms, Collision Resolution algorithms, QBD processes

\section{INTRODUCTION}

The deployment of high speed wireless LANs has become a topic of increasing interest over the past few years. The wireless environment raises some problems (e.g. multipath, interference, hidden terminal node, minimization of power consumption at the mobile terminals, handoff) that either are not present or have marginal importance in wired networks (Acampora 1986).

Wireless LANs usually have two types of realizations: infrastructured WLANs and ad-hoc WLANs. In infrastructured WLANs Time Division Multiple Ac-

*This work was supported by projects XUGA 10503 A96 and TIC95-0982-C02-01. Fundacion Caixa Galicia has supported D.V. Cortizo's stay at HUT, where part of the work was carried out. 
cess (TDMA) access control protocols are often preferred (Bantz et al. 1994). In ad-hoc WLANs mobile terminals establish peer-to-peer communication without the help of base stations and Carrier Sense Medium Access (CSMA) schemes may be advantageous because of their simplicity and because no station is compelled to assume special functions as in TDMA-based protocols. Unfortunately, random access protocols suffer from large access delay quantiles which make them unsuitable for delay-sensitive traffic support.

Modifications to the CSMA basic scheme have been proposed (Panwar et al. 1993) (Polyzos et al. 1987) to solve the instabilities of CSMA mechanisms and to improve the throughput-delay characteristics. The resemblance to the optimal FIFO discipline performance will be a trade-off with the overhead introduced and the protocol complexity.

The MAC protocol that we present in this paper is a collision resolution algorithm (Georgiadis et al. 1987) (Huang et al. 1986) aiming at the delay characteristics of a FIFO multiplexer. The protocol builds sets that contain the packets arriving (a packet arrival is defined to occur when a new packet is generated at any user site) within fixed-length grouping intervals. Packets belonging to the same set will be transmitted via CSMA but the different sets will be served following a FIFO discipline. The shorter the grouping time the closer to FIFO performance, but the signalling overhead will increase.

In the remainder of this section we present our assumptions on the system. In section II we describe the MAC protocol. In section III we describe both the simulation model and the analytical model developed to study the performance of the protocol. The results obtained are shown in section IV.

\subsection{Assumptions and description of the system}

- The network is supposed to be an ad-hoc high-speed wireless local area network. The possibility of handover has not been addressed.

- Either full connectivity among the stations or the existence of a higher level protocol to implement forwarding among nodes.

- Use of either radio or infrared channel providing a bit rate as seen by the MAC sublayer of $25 \mathrm{Mbps}$ (or higher).

- Every succesfully transmitted packet is positively acknowledged by the receiver via a short ACK packet. Multicast traffic has not been considered.

- We assume a constant packet length of aproximately $500 \mathrm{bits}$. This includes payload, overhead and the time needed to generate, transmit and process an ACK. Therefore all users in the system transmit packets of constant duration $T_{s} \approx 20 \mu s$. 
- The range of the network is supposed to be $R \approx 30 \mathrm{~m}$, so the maximum one-way propagation delay between any pair of users is $a \approx 0.1 \mu s$.

- We choose $T_{s}$ as the time slot length, and we further define mini-slots with length $t_{s}=3 a \approx 0.3 \mu s$. This way $T_{s}=N t_{s}$, where $N \approx 66$.

- The physical level will identify the different states of the channel: idle (no carrier), pilot tone transmission and packet transmission.

\section{DESCRIPTION OF THE PROTOCOL}

The basic medium access mechanism is CSMA with random backoff (an integer number of minislots) and adaptive backoff window size. However, this access mechanism is not applied to the whole set of stations having packets to transmit but only to a subset of them. The CSMA application to just some of the first arrived packets pending for transmission will allow to bring the protocol performance closer to the FIFO discipline behavior. Let us recall here the meaning of packet arrival: a packet arrival takes place whenever a packet is generated for transmission at any user site.

Our protocol will generate a collection of (possibly empty) Transmission Sets (TS) $\left\{S_{k}\right\}$, each of them containing the packets arrived during successive disjoint grouping intervals of constant length $W t_{s}$. These Transmission Sets are served (i.e. the associated packets are transmitted using CSMA) with a FIFO discipline, so that $S_{k}$ is served before $S_{j}$ if $k<j$. If every $S_{k}$ had no more than one packet to be transmitted we would be facing a FIFO-MAC algorithm, with packets being sent in order of arrival.

The number of packets per TS is closely related to the integer $W$, which determines the length of the grouping interval, and to the network load $\rho$. The smaller $W$ or $\rho$ are the more similar the behavior of the protocol will be to a FIFO system. On the contrary, the protocol will perform as pure CSMA if $W$ is made big enough. The value of $W$ will be a trade-off between the desired properties of a FIFO system and the protocol overhead.

Every active station keeps track of two sequence numbers SN associated to the TSs: the sequence number $N_{s}(t)$ of the TS in service and the sequence number of the TS that any packet arrival at time $t$ must join, $N_{g}(t)$.

The terminals shall include in the packet headers the sequence numbers $\left(N_{g}\left(t_{a}\right), N_{g}\left(t_{t x}\right)\right)$, where

- $t_{a}$ is the packet arrival time, $N_{g}\left(t_{a}\right)$ is the sequence number of the TS the packet belongs to, and

- assuming the packet starts being transmitted at $t_{t x}, N_{g}\left(t_{t x}\right)$ is the sequence number of the TS being generated by the time the packet attempts transmission. 
In the next subsections we will address the questions of how to generate, process and eliminate the transmission sets. For this sake the mobile stations will emit pilot tones of length one minislot, which also contribute to the protocol signalling overhead.

\subsection{Generation of the transmission sets}

Let us assume that the network has already been initialized and that no packets have been generated in the system for a long period of time. The system will be in idle state, characterized by all terminals in the network having $N_{s}(t)=N_{g}(t)$. When in this situation a packet is generated at any mobile node, it is immediately transmitted. Once the first transmission attempt takes place a busy period starts. During busy periods a new transmission set (either empty or not) is generated every $W$ minislots. TSs will be empty whenever there are no packet arrivals during the associated grouping intervals, but it is important to note that only during the busy periods empty TSs may be generated. Any packet arriving at $t_{1}$ will defer transmission until a time $t_{2}$ when its associated TS $N_{g}\left(t_{1}\right)$ begins to be served $\left(\left(N_{s}\left(t_{2}\right)=N_{g}\left(t_{1}\right)\right)\right.$. The service of a non empty TS consists of the successful transmission of all its packets via a scheduling which will be described in section 2.2.

If the network is stable the system must eventually get empty again, when $N_{s}(t)=N_{g}(t)$. At this point the generation of TSs is halted and the system enters an idle period. Packets finding at arrival time the system in idle state will directly attempt transmission, starting a new busy period, the generation of new TSs, and reinitiating the process described above.

\subsection{Service of the transmission sets}

The service of a Transmission Set consists of the successful transmission of all the packets that entered the system (at any station) during the grouping interval of length $W$ associated to the TS. The active stations must be able to identify not only the beginning and end of service of a non empty TS but also the empty TSs.

At the beginning of a TS service all the stations with packets belonging to that TS will emit a pilot tone during one minislot. This mechanism allows the stations to identify empty TSs (when there is no pilot tone present in the channel at the beginning of their service), discard them and proceed after only one minislot delay to the processing of the next TS. If the TS is non-empty after emitting the pilot tone the stations wait a random number of minislots before sensing the channel and, if idle, start transmission (CSMA with ran- 


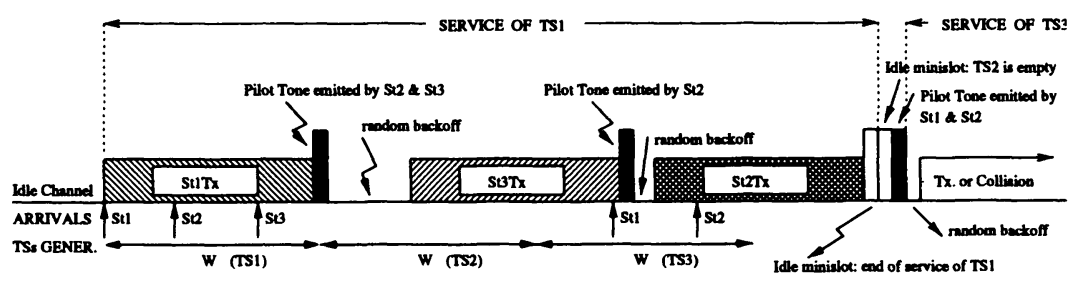

Figure 1 View of the operation of the protocol.

dom backoff window). At the end of the transmission there will have been either a collision or successful transmission, and the random backoff window value will be adapted accordingly.

After the successful transmission of a packet we may have two different scenarios: either there are still more packets in the TS that have not been transmitted (i.e. the service of the TS has not finished yet) or there are no more packets to transmit associated to the currently served TS, and the MAC should start processing the next TS.

These different situations can be easily identified by the active stations if we force all the terminals still having packets to transmit in the currently served TS to emit a pilot tone after every transmission. In the former case the stations waiting for the TS service to end will sense a pilot tone and keep on waiting. In the latter case, the terminals will sense an absence of carrier during one minislot, realize that the service of the $i$-TS has finished and start processing the $(i+1)$-TS.

In Figure 1 we have illustrated the operation of the protocol, assuming three Mobile Stations in the system St1, St2 and St3. When the system has been idle for a long enough period the first packet (St1 in the Figure) is immediately transmitted and it also starts the generation of TSs (TS1 in this case). After the transmission of the message (St1Tx) St2 and St3 emit a pilot tone, as they both have packets to transmit belonging to TS1. St3 gains access and after transmission of St3Tx, St2 emits a pilot tone, generates a random backoff and transmits its packet. As no mobile station has any other packet to transmit belonging to TS1 the next minislot remains idle, and the service of the next TS (TS2) starts. However, as there has not been any packet arrival during the interval associated to TS2, there will be another idle minislot indicating that TS2 is empty. In the next minislot the service of TS3 starts with a pilot tone generation by the stations having packets belonging to TS3 (St1 and St2).

\section{THE MODELS}

Our main objetive is to evaluate the density function of the delay suffered by a packet arriving in the system at an arbitrary point of time. This delay 


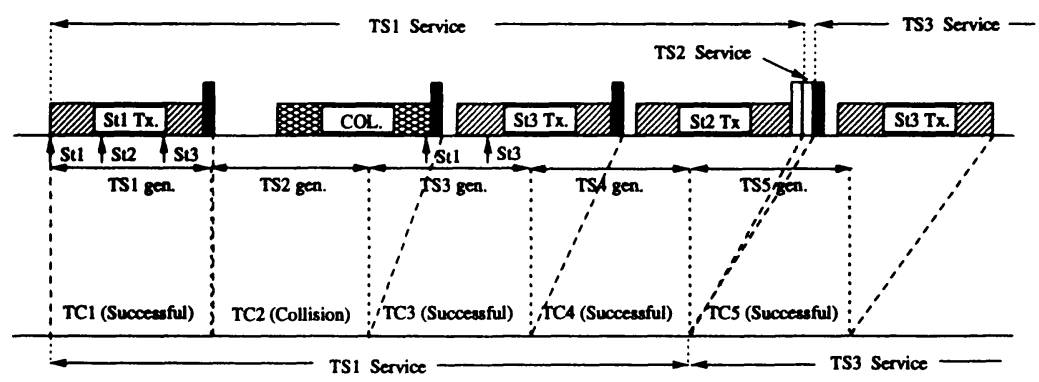

Figure 2 Synchronization achieved with the simplifications of the model.

density function will allow us to assess delay percentiles and therefore estimate the goodness of the protocol to support time-bounded services. We have developed both a simulation model and an approximate analytical model. For both models we have assumed that there are $m$ stations in the network and that the joint packet arrival process is Poisson with rate $\lambda$ (arrivals per slot $\left.T_{s}\right)$.

\subsection{Analytical model}

For the analytical model we have made the following simplifying assumptions:

- The minislot used for the pilot tones following every packet transmission is regarded as packet overhead.

- We will only consider the case where $W=N$, that is, the length of the grouping intervals is made equal to the length of the packets transmitted.

- The signalling minislot used at the beginning of every TSs (empty or not) service is neglected.

- The random backoff with adaptive window CSMA is replaced by a $p$ persistent CSMA. Adaptive mechanisms for the probability $p$ of transmission are not included in the analytical model.

- We will neglect the random backoff delay introduced by the stations before packet transmission.

- The maximum number of packet arrivals per Transmission Set has been bounded to a certain value $q_{\max }$.

In the simplified analytical model the system behaves as a TS-processing queue. During the busy periods we obtain a sequence of Transmission Cycles (TC) of length $W$. In every TC a packet transmission occurs (successful or not) and a new Transmission Set is generated (even if empty). When the system is idle neither TCs nor TSs are generated. It is the first next packet arrival who will reinitiate a busy period and the generation of both TSs and TCs. The simplifications synchronize the instants when packets get success- 
fully transmitted and the instants when new TSs are generated (see Figure 2).

The state of the system is defined by $(G, q)$, being $G$ the number of nonempty Transmission Sets pending to be served and $q$ the number of packets awaiting transmission in the TS in service. The pair $(G, q)$ can ve viewed as a Markov renewal stochastic process, and observing the state of the system at the end of every TC we get an embedded Markov Chain. The time elapsed between transitions will have a density function dependent on the state of the system (idle or busy). Being $u(t)$ the step function and $\delta(\cdot)$ the dirac delta function, we have

$$
\begin{aligned}
& f_{\text {busy }}(t)=\delta\left(t-W \cdot t_{s}\right) \\
& f_{\text {idle }}(t)=\frac{\lambda}{W} e^{-\omega^{\left(t-W \cdot t_{s}\right)}} u\left(t-W \cdot t_{s}\right) .
\end{aligned}
$$

\section{(a) System state probabilities and vector solution of the embedded markov chain}

The packet delay density function associated to the MAC protocol will be evaluated by conditioning on certain state of the system $(G, q)^{*}$. The values $\pi(G, q)^{*}$ represent the probabilities that a packet arrival finds that the last completed Transmission Cycle moved the system into state $(G, q)$. Note that $\pi(G, q)^{*}$ is not the probability that an arbitrary packet arrives being the state of the system $(G, q)$, but the probability that the packet finds that the last state transition of the embedded Markov chain was into the value $(G, q)$.

Previous to the assessment of the solution vector $\mathbf{v}$ of the embedded Markov chain (with one-step transition matrix $\mathbf{P}$ ) we will truncate the maximum value of $q$ to a certain value $q_{\max }$. If this bound is chosen large enough its influence on the accuracy of the results will be negligible.

Let us define the $\mathbf{M}_{q_{\max } \times q_{\max }}$ matrices $\mathbf{C}, \mathbf{D}$ and $\mathbf{E}$, as the matrices $\mathbf{F} \in$ $\mathbf{M}_{\left(q_{\max }+1\right) \times\left(q_{\max }+1\right)}, \mathbf{E}_{\mathbf{1}} \in \mathbf{M}_{q_{\max } \times\left(q_{\max }+1\right)}$ and $\mathbf{D}_{\mathbf{1}} \in \mathbf{M}_{\left(q_{\max }+1\right) \times q_{\max }}$

$$
\begin{array}{llll}
\mathbf{C}(q, r) & =P[(G, q) \rightarrow(G, r)] & G \geq 1 & q, r=1, \ldots, q_{\max } \\
\mathbf{D}(q, r) & =P[(G-1, q) \rightarrow(G, r)] & G \geq 1 & q, r=1, \ldots, q_{\max } \\
\mathbf{E}(q, r) & =P[(G, q) \rightarrow(G-1, r)] & G>1 \quad q, r=1, \ldots, q_{\max } \\
\mathbf{F}(q, r) & =P[(0, q) \rightarrow(0, r)] & q, r=0, \ldots, q_{\max } \\
\mathbf{D}_{1}(q, r)=P[(0, r) \rightarrow(1, q)] & q=1, \ldots, q_{\max } \quad r=0, \ldots, q_{\max } \\
\mathbf{E}_{\mathbf{1}}(q, r)=P[(1, q) \rightarrow(0, r)] & q=1, \ldots, q_{\max } \quad r=0, \ldots, q_{\max }
\end{array}
$$

The expressions for these transition probabilities are (only the nonzero probabilities are indicated) 


$$
\begin{aligned}
& \mathbf{F}^{0, i}= \begin{cases}e^{-\lambda} & i=0 \\
x(0, A) x(i, B)+\sum_{j=1}^{i-1} x(j, A) x(i-1-j, B) & i=1, \ldots, q_{\max }\end{cases} \\
& \mathbf{F}^{i, i}=e^{-\lambda} c(i, p) \quad i=1, \ldots, q_{\max } \\
& \mathbf{F}^{i, i-1}=e^{-\lambda} t(i, p) \quad i=1, \ldots, q_{\max } \\
& \mathbf{D}^{q, q}=\mathbf{D}_{\mathbf{1}}^{q, q}=\left(1-e^{-\lambda}\right) c(q, p) \\
& \left.\begin{array}{l}
\mathbf{D}^{q, q}=\mathbf{D}_{1}^{q, q}=\left(1-e^{-\lambda}\right) c(q, p) \\
\mathbf{C}^{q, q}=e^{-\lambda} c(q, p) \\
\mathbf{D}^{q, q-1}=\mathbf{D}_{1}^{q, q-1}=\left(1-e^{-\lambda}\right) t(q, p)
\end{array}\right\} q=2, \ldots, q_{\max } \\
& \mathrm{C}^{q, q-1}=e^{-\lambda} t(q, p) \\
& \mathbf{C}^{1, q}=x(q, \lambda) \\
& \left.\mathbf{E}^{1, q}=\mathbf{E}_{1}^{1, q}=e^{-\lambda} x(q, \lambda) /\left(1-e^{-\lambda}\right)\right\} q=1, \ldots, q_{\max } .
\end{aligned}
$$

Being $p$ the transmission probability in one minislot of the $p$-persistent CSMA, $A=\lambda / W, B=\lambda-A$ and

$$
\begin{aligned}
& x(i, \Delta)= \begin{cases}e^{-\Delta \Delta^{i}} & i=0, \ldots, q_{\max }-1 \\
1-\sum_{j=0}^{j=q_{\max }-1} x(j, \Delta) & i=q_{\max }\end{cases} \\
& t(i, p)=\frac{i p(1-p)^{i-1}}{1-(1-p)^{i}} \quad i=1, \ldots, q_{\max } \\
& c(i, p)=1-t(i, p) \quad i=1, \ldots, q_{\max }
\end{aligned}
$$

With the above definitions matrix $\mathbf{P}$ can be written as:

$$
\mathbf{P}=\left(\begin{array}{cccccc}
\mathbf{F} & \mathbf{D}_{1} & 0 & 0 & 0 & \cdots \\
\mathbf{E}_{\mathbf{1}} & \mathbf{C} & \mathbf{D} & 0 & 0 & \ldots \\
0 & \mathbf{E} & \mathbf{C} & \mathbf{D} & 0 & \ldots \\
0 & 0 & \mathbf{E} & \mathbf{C} & \mathbf{D} & \\
\vdots & \vdots & & \ddots & \ddots & \ddots
\end{array}\right)
$$

From the structure of $\mathbf{P}$ we see that it corresponds to a Quasy-Birth-andDeath (QBD) proccess. Therefore we will assess the vector solution $\mathbf{v}$ applying any of the existing algorithms (Neuts 1981), (Stewart 1994) to solve QBD markov chains. Once we have found the vector solution $\mathbf{v}$ for the embedded 
markov chain we make use of both the PASTA* property and the key Markov renewal theorem (Cinlar 1975) to calculate the state probabilities $\pi(G, q)^{*}$

$\pi(G, q)^{*}=\left\{\begin{array}{ll}\frac{\mathbf{v}(G, q)}{1+\mathbf{v}(0,0) / \lambda} & (G, q) \neq(0,0) \\ \frac{\mathbf{v}(0,0)}{1+\mathbf{v}(0,0) / \lambda}\left(1+\frac{1}{\lambda}\right) & (G, q)=(0,0)\end{array}\right.$.

These values will be needed to assess the transmission delay density functions of the the data packets. The derivation of these density functions is rather involved, and we have not included it in this paper (Cortizo et al. 1997).

\subsection{Simulation model}

Most of the simplifying assumptions of the analytical model are overcomed by the simulation model, whose main features are:

- The delay distribution function assessed includes the effect of both the pilot tones (signalling overhead) and the idle minislots associated to the random backoff in the CSMA algorithm.

- As in the analytical model, the random backoff with adaptive window CSMA is replaced by a $p$-persistent CSMA.

- The simulation model does not impose any constraint on the grouping interval length, allowing the study of the CDV percentiles vs. $W$.

\section{SIMULATIONS AND NUMERICAL RESULTS}

In this section we will analyze the behavior of the fixed-size random backoff window protocol, thus limiting the study to the non adaptive $p$-persistent CSMA case.

According to the assumptions of section 1.1, we have taken minislot lengths $t_{s}=0.3 \mu s$ and packet lengths $N=64$ minislots. The graphics obtained by simulation present the average of five experiments of length 64000000 minislots and the delay distribution functions $F_{D}(t)$ are depicted as $\log _{10}\left(1-F_{D}(t)\right)$.

Figure 3 shows, for a fixed load $\rho=0.6$, the protocol performance dependence on the transmission probability $p$, which is optimal for a value $p=0.1 / 0.2$. This value might seem surprisingly low but it must be noticed that the delays due to idle minislots (with $p=0.2$ a packet will be transmitted, in average, after a backoff of 5 minislots) are well paid off by the drastic re- 


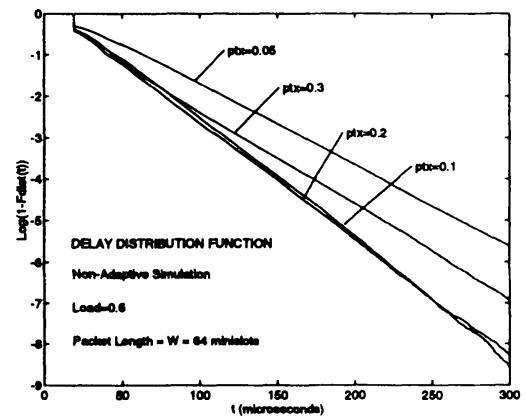

Figure 3 Determination of the optimal $p$.

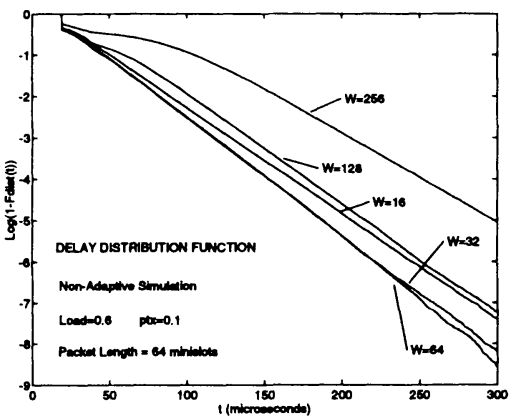

Figure 4 Determination of the optimal $W$.

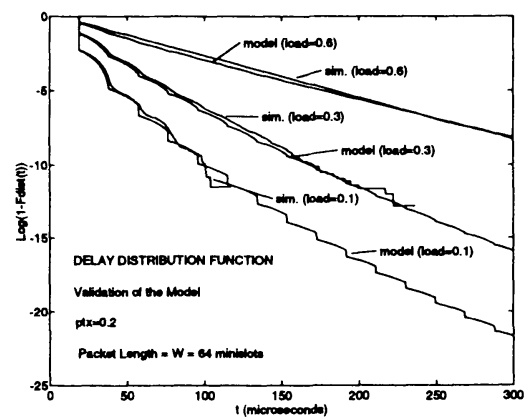

Figure 5 Validation of the model.

duction of the collision probability (each collision adds a delay of 64 minislots).

Figure 4 depicts the influence of the grouping interval length $W$ on the protocol performance. The value $W \approx N$ seems to be the best choice for load $\rho=0.6$. This result enhances the value of the analytical models, which only apply for this case. Moreover, the results corresponding to low-loaded systems $(\rho=0.1)$ lead to the same choices $p_{o p t}=0.2$ and $W_{\text {opt }} \approx N$ (Cortizo et al. 1997). For the remainder of the section we will therefore assume $W=N$.

Figure 5 validates the analytical model by comparison with the results obtained by simulation. The model gives always similar results to those obtained by simulation. For lower values of $p$ (less than 0.1 ) we expect an impairment in the behavior of the analytical approach, as it neglects the idle minislots introduced by the random backoff windows.

Figures 6 and 7 show the $10^{-3}$ and $10^{-6}$ packet delay quantiles for different network loads. The quantiles are defined as the delays exceeded with less probability mass than $10^{-3}$ and $10^{-6}$ respectively. From these quantiles one 
can easily find estimations of the Cell Delay Variation introduced by the access protocol (Roberts et al. 1996). The graphics show the good behavior of the MAC protocol, allowing the support of real-time services with loads close to 0.8 .

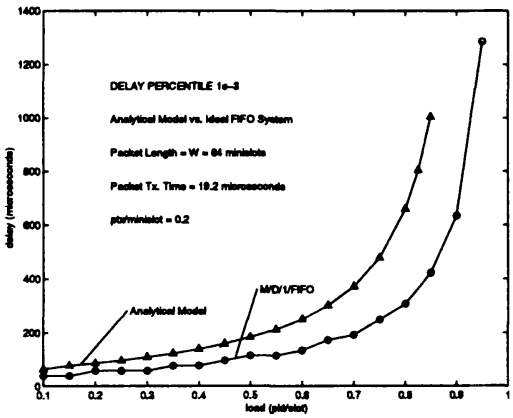

Figure $610^{-3}$ quantile for the packet delay.

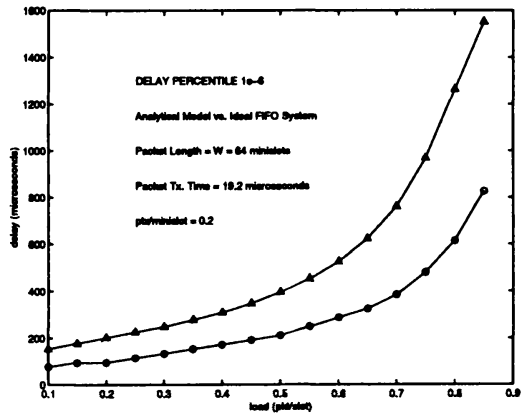

Figure $710^{-6}$ quantile for the packet delay.

\section{CONCLUSIONS}

In this paper we have presented a MAC protocol oriented to ad-hoc Wireless LANs. This protocol is a Group Resolution Algorithm, as it gathers into Transmission Sets the packet arrivals taking place in grouping intervals of fixed length. Every Transmission Set is served via an adaptive-window random backoff CSMA, and the different TSs are handled with FIFO discipline.

The protocol performance lies between that of CSMA and FIFO algorithms, depending on the length of the grouping intervals. The shorter these are, there will be less packets in average per TS and the MAC will behave as a distributed FIFO scheduling. However, the signalling overhead introduced by the protocol grows when the grouping interval length decreases.

We have developed a simplified analytical model which only considers the situation where the packet lengths equal the size of the grouping intervals. The results obtained show that the protocol can support real-time services even when the network load is moderately high (loads up to 0.8 ).

\section{REFERENCES}

Acampora, A. (1996) Wireless ATM: A Perspective on Issues and Prospects. IEEE Personal Communications, Vol. 3, No. 4, August 1996, 8-17. 
Bantz, D.F. and Bauchot, F.J. (1994) Wireless LAN Design Alternatives. IEEE Network, March/April 1994, 43-53.

Roberts, R., Mocci, U. and Virtamo, J. (1996) Broadband Network Traffic, Performance Evaluation and Design of Broadband Multiservice Networks Final Report of Action COST 242. Springer-Verlag, Berlin.

Cinlar, E. (1975) Introduction to Stochastic Processes. Ed. Englewood Cliffs, New Jersey.

Georgiadis, L., Papantoni-Kazakos, P. (1987) A 0.487 Throughput Limited Sensing Algorithm. IEEE Transactions on Information Theory, Vol. IT-33, No. 2, March 1987, 233-237.

Huang, J.C. and Berger, T. (1986) Delay Analysis of 0.487 Contention Resolution Algorithms. IEEE Transactions on Communications, Vol. COM34, No. 9, September 1986, 916-926.

Marcel F. Neuts, M.F. (1981) Matrix-Geometric Solutions in Stochastic Models, An Algorithmic Approach. The John Hopkins University Press, Baltimore.

Panwar, S.S., Towsley, D. and Armoni, Y. (1993) Collision Resolution Algorithms for a Time-Constrained Multiaccess Channel. IEEE Transactions on Communications, Vol. 41, No. 7, July 1993, 1023-1026.

Polyzos, G.C., Molle, M.L. and Venetsanopoulos, A.N. (1987) Performance Analysis of Finite Nonhomogeneous Population Tree Conflict Resolution Algorithms Using Constant Size Window Access. IEEE Transactions on Communications, Vol. COM-35, No. 11, November 1987, $1124-1138$.

Stewart, W.J. (1994) Introduction to the Numerical Solution of Markov Chains. Princeton University Press.

Cortizo, D.V. and García, J. (1997) A MAC Protocol for Ad-Hoc Wireless ATM LANs. Departamento Electrónica e Sistemas, UDC, Internal report, Aug. 1997. 